\title{
Malnutrición intrauterina: comparación de curvas de crecimiento en la detección de alto riesgo neonatal
}

\author{
Germán Mühlhausen M.1; Carlos Navarrete G.1 \\ Intrauterine malnutrition: detection of neonatal high risk \\ by comparing intrauterine growth curves
}

\begin{abstract}
To evoluale the clinical usefulnes of Lreign (Lubchenco) ond a local iluez, Lucero, Venturajuncai inirauterine growth standards in the search of high risk infants from low bisth weight newborns, bolth standards were used in 8989 newborn infants from nolthweslern metropoliton Sanliggo, Chile. The local standard identified 753 infants with intrauterine growth retardation |IUGR\} whlle the toreign one detected only 270 such infonts. Among 482 neonoles with growth retardalion by the local standards, which included 194 UUKR also selected by the Lubchenco's criteriae, there were no significant internal diferences on risks for hospilalization, deliveries by cesarean section (32.8\%), hipoglycemia (3.5\%) and policythemia [17.8\%, p>0.05, but these risks were significontly higher than in 5024 newborn infants with adecuate weight for gestaticnal $\mathrm{age}$ in which that information was readily available $120 \%, 0.3 \%$ and $0.3 \%$ respectivelyl.
\end{abstract}

(Key wordss inlant newborn, intauterine growth relordation, intrauterine growh curves).

A medida que la mortalidad infantil en Chile se ha reducido a lo largo de los años, la mortalidad neonatal, si bien menos en términos absolutos, ha ido cobrando mayor importancia porcentual dentro del total',2. En su disminución ha tenido importante participación la organización de sistemas más adecuados de cuidados intensivos neonatales a partir del año $1980^{3}$. Sin embargo, como en otros paises de mayor desarrollo relativo, la mortalidad del primer día de vida se ha hecho más relevante4.

El retardo del crecimiento intrauterino (RCIU), sigue siendo una importante causa de morbilidad, hospitalización y mortalidad a pesar de ser una entidad perfectamente reconocible, casi siempre susceptible de diagnosticar y, en cierta medida, de tratar en el período in trauterino cuando se cuenta con un buen control obstétrico, lo cual, no siempre se cumple 5 -13.

En nuestro hospital en el período que incluye los años 1989 y 1990 , nacieron 8989 niños, de los cuales $270(3,0 \%)$ tenían evidencia de RCIU

1. Unidad de Recién Nacidos, Servicio de Pediatria, Hospital Fétix Buines Cerda. Trabajo presentado en Ia XIV Jomadas Chilenas de Pediatría 1991. de acuerdo con la curva de crecimiento prenatal de Lubchenco ${ }^{14}$. Este número tan exiguo guarda relación con las dificultades que entraña el diagnóstico de RCIU del recién nacido, entre las cuales una de las principales, ha sido la falta de curvas nacionales, suplida por muchos ax́os con curvas extranjeras, especialmente la de Lubchenco, hecho que ha sido muy criticado ${ }^{14.16}$. Empero hace poco tiempo se han publicado curvas hechas en Chile ${ }^{17-23}$, siendo la más reciente la de Juez, Lucero y Ventura-Iuncá23, que podría relacionarse en forma más fidedigna con la realidad antropométrica de los RN chilenos ${ }^{17}+18,23$.

El objetivo de nuestro estudio fue comparar dos curvas (una estadinense, la de Lubchenco ${ }^{14}$, y otra nacional, Ja de Juez, Lucero y Ventura-Jun$c^{23}$ ) de crecimiento intrauterino en nuestra población de recién nacidos, tratando de detectar eventuales diferencias en su capacidad de identificar recién nacidos con RCIU; averiguar si tales diferencias, si existían, implicaban selecciớn de poblaciones significativamente diferentes, con trascendencia clínica o epidemiológica y si, finalmente, la pertenencia a alguna de estas poblaciones estaba o no asociada a la presencia de determinadas afecciones. 


\section{Material y Método}

De un total de 8989 niños nacidos en la Matemidad del Hospital Félix Bulnes Cerda, durante el periodo comprendito entre el 1 de mayo de 1989 y el 30 de abril de 1990,753 $(8,4 \%)$ eran pequeños para la edad gestacional (RNPEG), según la curva de Juez, Lucero, Ventura-Juncł y $270(3,0 \%)$ según la estadinense (menores al percentil $10 \mathrm{de}$ ambas curvas en cada caso). Por diversas razones como pérdida de muestras de sangre, negativa de la madre, Iraslado a otro hospital, altas precoces o sin aviso, solo 482 de los RNPEG seleccionados por el criterio nacional podieron ser estudiados, lo que se realizó mediante la aplicación prospectiva de un protocolo de seguimiento durante su estadia, desde el nacimiento hasta su alta de la unidad de puerperio o la de recién nacidos. El registro incluyó vía del parto; Apgar a 1 min y $5 \mathrm{~min}$ de nacer; edsd, paridad y enfermedad matemas; peso, talla, circunferencia crancana y evolución intrahospitalaria del reçién nacido en caso de enfermedad de éste, incluyendo resultados de los exámenes de laboratorio, todo to cual se obtuyo de una ficha individual y se proces 6 posteriormente en forma computacional para su análisis.

La edad gestacional de los RV fue determinada por la fecha de la óltima regla (FUR) y, o, ecografía obstétrica cuando ésta existía. La evaluación pediátrica de la edad gestacional se efectuó de acuerdo a Usher y Dubowitz ${ }^{24,21}$.

Por definición los 482 RNPEG seleccionados estaban bajo el percentil 10 de la curva nacional y ellos constituyeron el grupo 1 de este estudio. Entre esos mismos había 194 niños cayo peso era inferior al percentil 10 de Lubchenco, los que formaron el grupo 216, 18. Los dos grupos de RNPEG fueron comparados entre sí y con $5024 \mathrm{RN}$ de peso adecuzdo a la edad gestacional ( $A E G$ ).

A todos los RN estudiados se les efectuó hematocrio central, glícemia a las 4 horas de vida y seguimiento durante la totalidad de su estadía hospitalaria (puerperio y, o, unidad de neonatología) hasta el alta, con el fin de pesquisar la aparición de manifestaciones de enfemedad to. 13, 26-29. Se estudió el tipo de parto, la existencia de Apgar bajo (menor o igual a 3 al minulo 1 y, o, a los cinco minutos), tasa de hospitalizacion, incidencia de ictericia patológica (que necesiló foloterapia), incidencia de poliglobulja (Hto $>65 \%$ ) e hipoglicemia (glicemia < $40 \mathrm{mg} \%$ ) tomando en considera ción la persistencia de la alteración en dos exámenes sucesivos.

E1 análisis estadístico efectuado con la prueba de Chi cuadrado, considerándose significativa una diferencia cuando $p$ resultó infecior $\star 0,05$. Además se usó la relación de disparidad ("odds ratio") como nétodo descriptivo y, como medida de dispersión, intervalos de confianza de $95 \%$, con el fiл de predecir el riesgo relativo usando también una significación estadistica de $\mathrm{p}<\mathrm{a} 0,05$.

\section{Resultados}

Al aplicar la curva nacional aparecieron 288 recién nacidos con retardo del crecimicrto intrauterino, en exceso que con el criterio de Lubchenco, diferencia que resultó estadísticamente significativa (figura 1). Las incidencias de parto eutócico $(63,3$ y $57,7 \%)$ y por ceśrea $(32,8$ y $37,1 \%$ ) en los grupos 1 y 2 fueron similares, pero ambas eran significativamente distintas que las observadas en RNAEG, donde los partos eutócicos representaron $71,8 \%$ y las cesárcas $26,0 \%$ $(\mathrm{p}<0,05)$. El riesgo relativo de parto por cesárea resultó, en los dos grupos de RNPEG, mayor que $1: 1,3$ veces con respecto a los RNAEG.

Las incidencias de Apgar $\leq 3$ en el primer minuto fueron $2,3 \%$ (n: 11 ) en el grupo $1,4,6 \%$ (n: 9) en el grupo 2 y $1,7 \%$ (n: 116) en los RNAEG, y $0,4 \%$ (n: 2), $1 \%$ (n: 2 ) y $0,4 \%$ (n: 26 ), respectivamente, a los $5 \mathrm{~min}$., diferencias que no fueron estadísticamente significativas excepto al minuto entre los niños del grupo 2 y los RNAEG $(p<0,05)$.

La frecuencia de hospitalización se comparo con la del total de $8989 \mathrm{RN}$ dcl anto estudiado, donde fue de $12,9 \%$ (1 $164 \mathrm{RN}$ ), la del grupo 1: $24,1 \%$ (116 RN).y el grupo $2: 28,9 \%$ (56 RN),

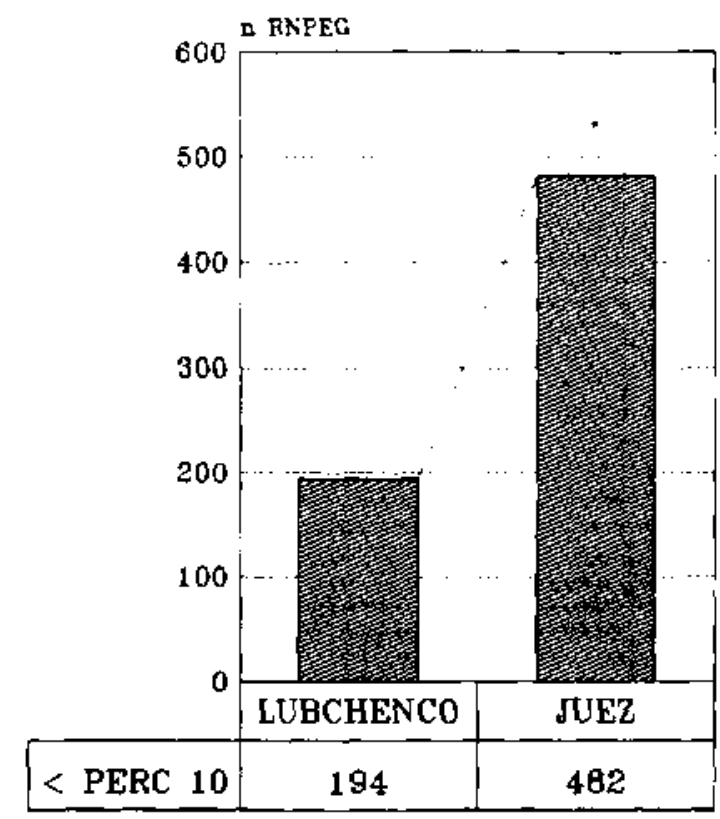

TIPO DE CURVA

- $=\mathbf{P}<0,05$

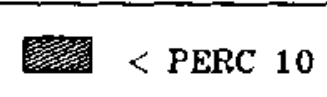

Figura 1: Describe el número de RNPEG de acuerdo al tipo de carva de crecimiento intraterino. 


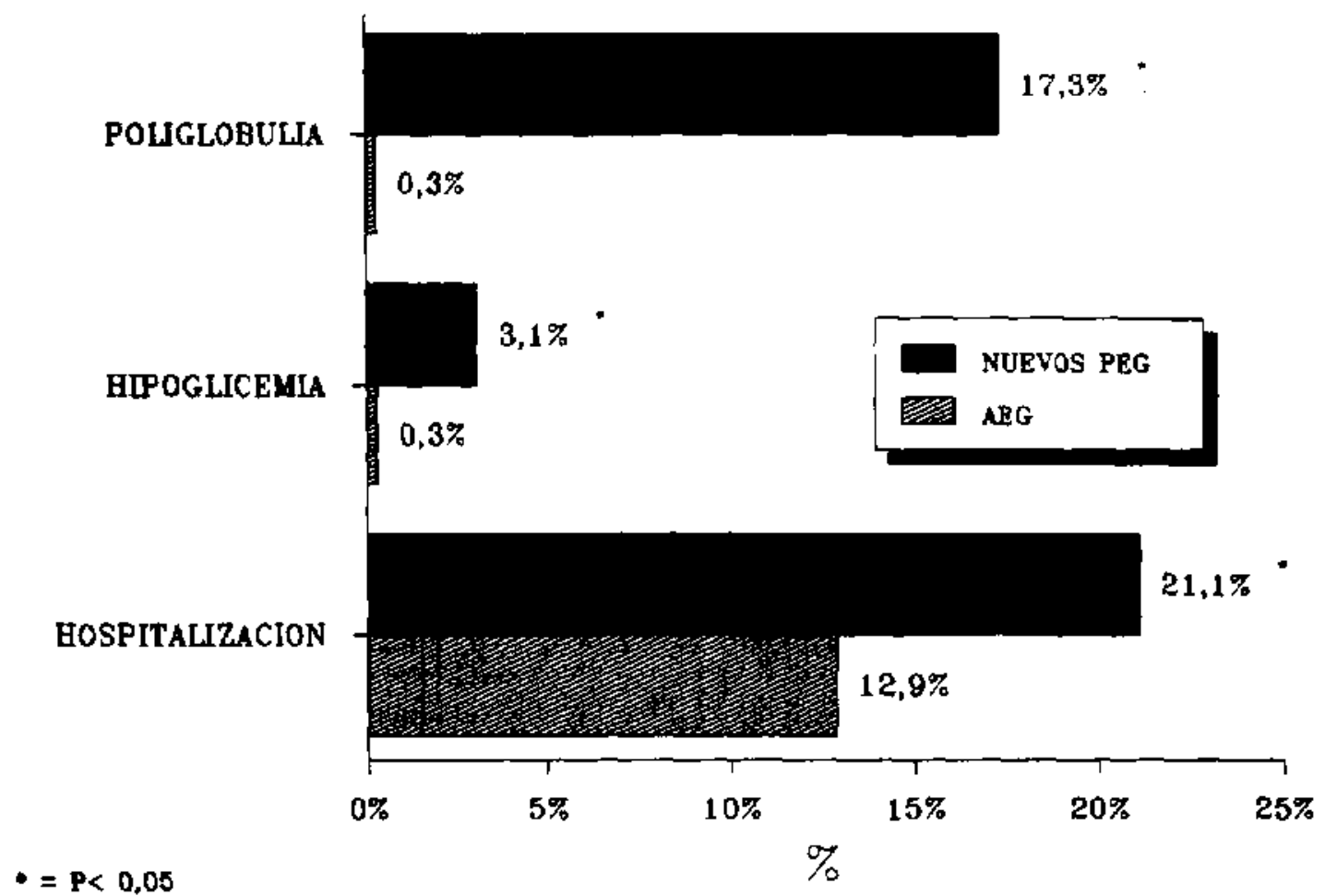

Flgura 2: Muestra la incidencia de diferentes entidades clínicas de los "nuevos PEG" comparados con los RNAEG.

siendo significativamente distinta $(\mathrm{p}<0,05)$ entre el total y los grupos 1 y 2 , pero no entre estos últimos, cuyo riesgo relativo de hospitalización fue mayor de 1:2,1 veces que el del total.

Las frecuencias de hipoglicemia fueron $3,5 \%$ en el grupo $1 ; 4,1 \%$ en el grupo 2 (p NS) y $0,3 \%$ en los RNAEG ( $p<0,05$ ). Las de poliglobulia $17,8 \%, 18,6 \%$ (p NS) y $0,3 \%$ (p<0,05) y las de ictericia $6,6 \%, 7,2 \%$ (p NS) y $7,4 \%$ (p NS), respectivamente. El riesgo relativo de tener hipoglicemia o poliglobulia en ambos grupos RCIU fue 1:12 o mayor que en los RNAEG. El riesgo relativo de tener alguna de las entidades clínicas anteriormente citadas, comparando los percentiles 10 de la curva nacional con el percentil 10 de la estadinense, no fue estadísticamente mayor en ninguna de las dos curvas.

Con el fin de poder determinar si los 288 nuevos RNPEG resuliantes de la aplicación de la curva nacional constituían una población diferente, se compararon con el grupo do RNAEG, sin encontrar diferencias significativas con res- pecto al tipo de parto, incidencia de Apgar bajoe ictericia patológica, pero sí las había en cuanto a poliglobulina, hipoglicemia y tasa de hospitalización $(p<0,01)$ (figura 2$)$, con un riesgo relativo de presentar -este subgrupo-hipoglicemia y poliglobulia, mayor a 1:11 veces que entre los RNAEG. En cuanto a la tasa de hospitalización, ésta fue mayor a 1:1,8 veces que la de los RNAEG.

\section{Comentario}

En diversas publicaciones se ha señalado la importancia de disponer, en cada pais, de curvas de crecimiento intrauterino con el fjn de evaluar a sus propios niños ${ }^{15,18-23}$. Los resultados descritos señalan que, al usar el percentil 10 de la curva esiadinense, una importante cantidad de RNPEG no fueron detectados en este medio, los que, en cambio, aparecían (nuevos RNPEG) si se usaba la nacional, al igual que en la publicación original ${ }^{18}$. Estos correspondían casi con exactitud al 
nümero de RNPEG (288) que se obtenian al utilizar conno límite superior el percentil 25 en lugar del 10 de la curva estadinense. Al encontrarse diferencias significativas entre este grupo de "nuevos RNPEG" con los RNAEG se demostró que el mayor númeno de niños que captaba la curva nacional, correspondía a RN con mayor ricsgo de morbilidad y hospitalizacion que los RNAEG. Lo anterior resuelve la inquietud señalada en una comunicación nacional ${ }^{30}$, que plantcaba que usar la curva de Juez, Lucero, VenturaJuncá podría aumentar cl gasto en los servicios de salud al tener que someter a exámenes preventivos un número mayor de $\mathrm{RN}$, sin que necesariamente todos éstos fueran a tener mayor riesgo de enfermedad y hospitalización.

Por otro lado, si bien los percentiles 10 de ambas curvas resultan buenos predictores de diversas situaciones de riesgo, como tipo de parto, Apgar bajo, tasa de hospitalización y entidades clínicas como hipoglicemia y poliglobulia, sin diferencias en las proporciones en que las detectan, el criterio nacional tiene la ventaja de identilicar un nímero significativamente mayor de ninos en riesgo de enfermar, por lo que parece conveniente preferir, en Chile, su empleo sobre su altemativa estadinense.

\section{Resumen}

Se estudiaron prospectivamente 8989 recién nacidos, en busca de pacientes con retardo del crecimiento intrauterino (RCIU) de acuerdo a dos curvas de crecimiento intrauterino, la estadinense, de Lubchenco y la local de Juez, Lucero, Vcntura-Juncá. Este último criterio permitiớ detectar 753 recién nacidos con RCIU en comparación con el estadinense, que identificó sólo 270. Se encontró mayor riesgo de hospitalización, cesáreas, apgar bajo y mayor incidencia de hìpoglicemia y poliglobulia en los recién nacidos scleccionados como RCIU por ambas curvas que en los 5024 recién nacidos de peso adecuado para la edad gestacional en que también se regis. traron dichas determinaciones. Lo anterior implica que la curva local detecta mayor número de casos en riesgo y, por lo tanto, es de mayor utilidad en cste medio.

(Palabras clave: recién nacido, retardo de crecimiento intrauterino, curvas de crecimiento intrauterino.)

\section{Agradecimientos}

Los autores del trabajo agradecen la yaliosísima colaboración prestada por los médicos de la Unidad de Recién Nacidos, como así, la de las matronas de Recién Nacido Inmediato، en especial las Sras. Magaly Burdiles, Ana María Rivera y Marlene Allaein.

\section{Referencias}

1. Vargas N, Quezada L: Mortalidad infantil: Análisis de tendencias recientes e identificación de regiones y causas de ricsgo mayor. Rev Chil Pediatr 1988; 59: 122-128.

2. Puffer $R$, Serrano $C$ : Características del peso al nacer. Boletín de Ia Organización Panamericana de la Salud, 1988; 24-29.

3. Ferreiro $M$, Beca $J P$, Maler $R$, Vaisman $S$ : Implementación en la atención neonatal en Chile. Pediatría (Saniago) 1976; 19: 282-284.

4. Kramer MS: Intrauterine growth and gestational duration determinants. Pediatrics 1987; 80:502-511.

5. Aries $F$ : The diagnosis and management of intrauterine growth retardationt. Obstet Gymecol 1977; 49: 293-298.

6. Belizán J; Villar J; Nardin J es al.: Diagnosis of intrauterine growh retardation by a simple clinical method: Measuremenl of uterine height. Am J Obsiel Gynecol 1978; 131 : 643-646.

7. Villar $J$, Belizán $J$ : The relative contribution of prematurity and fetal growth retardalion to low birth weight in developing and developed societies. Am I Obstet Gynecol 1982; 143: 793-798.

8. Belizan J, Lechig A, Villar J: Distribution of low-birth weight babies in developing countries. An I Obstet Gynecal 1978; 132: 704-705.

9. Campbell S, Thoms A: Ulirasound measurement of the fetal head to abdomen circunference ratio in the assessment of growlh relardation. Br J Obstet Gynaecol 1977; 84: 165-174.

10. Eaves $L C$, Nu!toll JC, Klonoff $H$ et al.: Developmettal and phsycological tes1 scores in children of lowi-birth weight. Pediatrics 1970: 45:9.20.

11. Low JA, Galbraith RS, Muir D et al,: Intrauterine growth retardation: A study of long term morbidity. Am J Obster Gynecol 1982; 142: 670-677.

12. Simpson $G F$ and Creasy $R K$ : Obstervic management of the growth retarded baby. Clin Obstet Gynaecol 1984; 11: $481-497$.

13. Starfield $B$, Shapiro S., HcCormick $M$ et at.: Mortality and morbidity in infants with intrauterine growth reLardation. J Pedialr 1982; 101: 978-983.

14. Lubchenco LO, Haneman C. Dressler $N$ et al.: Intra. uterine growth estimated from livebirths at gestational age from 26-42 wecks. Pediatrics 1963; 32:793-800.

15. Milier HC. Hassanein $\boldsymbol{X}$ : Diagnosis of impaired fetal growth in newbom infants. Pediatrics $1971 ; 48: 511$. 522.

16. Usher RH and $M C L e a n ~ F H$ : Intrauterine growth of liveborn Caucasian infants at sen level: Standards obtained from measurements in 7 dimensions of infants born between 25 and 42 weeks of gestation. J Pediatr 1969 ; 74: $901+910$. 
17. Juez $G$, Venturo-Jancó $P_{s}$ Lucero $E$ - Crecimiento iftranterino en un grupo seleccionado de recién nacidos chilenos. Rev Med Chile 1984; 112: 759-764.

18. Ventura Jutcd $P$, Juez $G, L$ ucero $E$ : Desnutrición inIsauterina: Identificación de una nueva población de ailo riesgo con una curva de peso chilena. Rev Med Chile 1986; 114: 790-797.

19. Rizzardini $M$, Silva $E$, Schidlow $D$ : Curva de crecimiento intsauterino en recién nacidos chiletros. Rev Chil Pediatr 1972; 43: 7-13.

20. Alvear J, Beca JP, Saquedra $G$ et al: Evaluación del crecimiento íntrauterino del recién nacido. Rev Chil Pediatr 1983; $54:$ 374-378.

21. Weidt E, Rosselot $S$, Tohd $D$, Ardrade $C$. Evaluación de crecimiento intraulcrino mediante el peso de nacimiento. Rev Chil Pediatr 1988; 59: 267-269.

22. Beca JP, Rizarardini $M$, Weidt E el al: Talla, pesoy perimetro craneano según edad gestacional en recién nacidos de menos de 35 semanas. Rev Ohil Pediatr 1989; 60: 1-5.

23. Juez $G$, Lucero $E$, Ventura-Juncd $P$ et al.: Crecimiento intrauterino en recién nacidos chilenos de clase media. Rev Chil Pediatr 1989; 60: 198-202.
24. Dubawifz LMS, Dubowitz Y. Goldberg C: Clinical as sessment of gestational age in the newborn infant. J Pediatr $1970 ; 77: 1-10$.

25. Uster $R, M$ dean $F$, Scott $K E$ : Iudgcmest of fetal age. $\Pi$. Clinical significance of gestational age and an objective method of its assessment. Pedialr Clin North Am 1966; 13: 835-848.

26. Oh $W$ : Neonatal polycythemia and hyperviscosily. Ped Clin North Am 1986; 33: 523-532.

27. Rivera W, Sónchez l, Cavagnaro F et al.: Poliglobulja neonatal. Revisión de 110 casos. Rev Chil Pediatr 1988; 59: 257-260.

28. Miranda LE, Dueck HS: Perinatal glucose homeostasis: The unique character of hyperglyecmia and hypoglycemia in infants of very low birth weight. Clin Perinatol $1977 ; 4: 351-365$.

29. Ogola ES: Carbohydrate metabolism in the fetus and nconate and altered neonatal glucoregulation. Pediatr Clin North A.m 1986; 33: 25-45.

30. Aspillaga $C$. Bianchi $\mathcal{R}_{\text {.: }}$ Observaciones referentes a la curva de crecimiento intrauterino difundida por el Ministerio de Salud. Rev Chil Pediatr 1992; 63:60-62. 